\title{
Axiomatizations for Temporal Epistemic Logic with Perfect Recall over Linear Time
}

\author{
Szabolcs Mikulás \\ School of Computer Science and Information Systems \\ Birkbeck College \\ London, U.K. \\ szabolcs@dcs.bbk.ac.uk
}

\author{
Mark Reynolds and Tim French \\ School of Computer Science and Software Engineering \\ University of Western Australia \\ Perth, Australia \\ \{mark,tim\}@csse.uwa.edu.au
}

\begin{abstract}
This paper presents various semantic interpretations for logics of knowledge and time with prefect recall. We allow both past and future operators and examine the interpretation of different linear flows of time. In particular, we present temporal epistemic logics for each of the following flows of time: arbitrary linear orders; the integers; the rationals; the reals; and for uniform flows of time. (By uniform flows of time, we mean that time is an arbitrary linear order that is common knowledge to all agents). We propose axiomatizations for all logics except the last case, for which we show that no finite axiomatization can be found. The axiomatizations are shown to be sound and complete in the case of arbitrary linear orders and the rationals.
\end{abstract}

Keywords: epistemic logic, temporal logic, complete axiomatizations

\section{INTRODUCTION}

Logics of time and logics of knowledge have, independently, found many applications in reasoning about computational systems. Temporal logics are applied to reason about program correctness [12], and logics of knowledge are applied to reason about information in distributed systems [4]. Furthermore, the combination of the two are well-studied and applied for reasoning about security protocols [9].

The application of temporal epistemic logic to specifying and verifying computational systems have generally relied on discrete flows of time [10], [5], and interactions between logics of knowledge and non-discrete flows of time have not received a great deal of attention in the literature. Independent of epistemic logic, there has been a detailed study of temporal logic over various flows of time [14], [1]. In [6] some combinations between epistemic logic and non-discrete temporal logics were examined. This work presented the complexity of a number of temporal epistemic logics and derived axiomatizations for some simple cases not involving perfect recall. Here we seek to further this vein of research by providing axiomatizations for temporal epistemic logics with perfect recall given flows of time defined over different linear orders including: discrete linear orders (the integers), dense linear orders (the rationals) and continuous linear orders (the reals). As the foundation of these results we present an axiomatization of temporal epistemic logic over arbitrary linear orders (so that the agents may not know the flow of time). We show that strengthening this class so that the agents always know the flow of time results in a logic that cannot be finitely axiomatized. For the interactions between knowledge and time we assume perfect recall (or no forgetting [10]), so that once an agent knows something about the set of possible flows of time, they always know it.

Our extensions to non-discrete flows of time are not only motivated by aesthetic arguments: There are numerous applications where we are required to not only reason about a computation, but also it's interaction with the environment. The study of hybrid systems allows models of discrete computation (automata) to interact with continuous systems modeled by differential equations [3]. There is an emerging application for formal models of dense and continuous systems. Complete theories for temporal epistemic logics over non-discrete time may complement such applications.

\section{The language}

Let the natural number $n$ be fixed. We will investigate the following logic $\mathcal{T} \mathcal{E} \mathcal{L}_{n}$. The language consists of the propositional connectives, the epistemic modalities $\mathrm{K}_{i}$ (for $i \in n$ ) and the temporal operators $\mathrm{F}$ and $\mathrm{P}$. The set Fml of formulas is built up from a set $P$ of atomic propositions in the usual manner.

Semantics is given as follows. Let $S$ be a set of (local) states and $\mathcal{L}$ be a set of linear flows of time $(L,<)$. A run $r$ over $\mathcal{L}$ is a function $r: L \rightarrow{ }^{n} S$ for some $(L,<) \in \mathcal{L}$, i.e., for a given linear flow $(L,<)$, it associates an $n$-tuple of states with every time point in $L$. For runs $r, r^{\prime}$ over $\mathcal{L}$, we will write $r(l) \sim_{i} r^{\prime}\left(l^{\prime}\right)$ (for $i \in n$ ) iff $r(l)_{i}=r^{\prime}\left(l^{\prime}\right)_{i}$. A valuation $v$ is function $v: P \rightarrow \mathcal{P}\left({ }^{n} S\right)$ associating to every atomic proposition in $P$ a set of $n$-tuples of states. A model $\mathcal{M}$ consists of a set $R$ of runs over $\mathcal{L}$ and a valuation. Truth of a formula is evaluated at a point $l$ of a run $r$. The nonpropositional cases are as follows: for $i \in n$,

- $(\mathcal{M}, r, l) \models \mathrm{K}_{i} \varphi$ if and only if $\left(\mathcal{M}, r^{\prime}, l^{\prime}\right) \models \varphi$ for all $r^{\prime}$ and $l^{\prime}$ such that $r(l) \sim_{i} r^{\prime}\left(l^{\prime}\right)$

- $(\mathcal{M}, r, l) \models \mathrm{F} \varphi$ if and only if $\left(\mathcal{M}, r, l^{\prime}\right) \models \varphi$ for some $l^{\prime}$ such that $l<l^{\prime}$

- $(\mathcal{M}, r, l) \models \mathrm{P} \varphi$ if and only if $\left(\mathcal{M}, r, l^{\prime}\right) \models \varphi$ for some $l^{\prime}$ such that $l^{\prime}<l$

We will use the usual abbreviations: $\mathrm{L}_{i} \varphi$ for $\neg \mathrm{K}_{i} \neg \varphi, \mathrm{G} \varphi$ for $\neg \mathrm{F} \neg \varphi$, and $\mathrm{H} \varphi$ for $\neg \mathrm{P} \neg \varphi$. For a temporal operator $\mathrm{T}$, let 
$\mathrm{T}^{\prime}$ denote its "weak" version (defined by $\leq$ instead of $<$ ), e.g., $\mathrm{P}^{\prime} \varphi$ is defined as $\mathrm{P} \varphi \vee \varphi$.

\section{Perfect recall}

The logic of perfect recall is defined by the following semantical condition: for all $i \in n$,

$$
\begin{aligned}
& \text { if } r(l) \sim_{i} r^{\prime}\left(l^{\prime}\right) \text { and } k<l \text {, then there is } k^{\prime} \leq l^{\prime} \text { with } \\
& \qquad r(k) \sim_{i} r^{\prime}\left(k^{\prime}\right) .
\end{aligned}
$$

Let $\mathcal{T} \mathcal{E} \mathcal{L}_{n}^{p r}$ denote the logic $\mathcal{T} \mathcal{E} \mathcal{L}_{n}$ satisfying the additional condition of perfect recall.

This simple definition for perfect recall is very intuitive in the case where all agents knowledge is synchronized (that is, for all runs $r$, for all distinct points $x$ and $y, r(x) \chi_{i} r(y)$, see [4]). However, here we do not restrict the models to be synchronized and this can lead to some unintuitive models. For example, consider the model consisting of a single run over the integers, and a single agent such that for all $x, y \in \mathbb{Z}$, $r(x) \sim r(y)$ if and only if $x$ and $y$ have the same parity. Such an agent is unable to distinguish between the past, present and future, which seems contrary to notion of perfect recall.

From the perspective of logic we are mostly interested in the properties that we are able to express using formulae, rather than anomalous classes of models. Some interesting examples of such properties are:

1) An agent cannot distinguish between a single point in one run and an infinite set of points (an interval) in another run. Only in such a case would the following formula be satisfied:

$$
\mathrm{K}_{i} x \wedge \mathrm{HK}_{i} \neg x \wedge \mathrm{L}_{i}(x \wedge \mathrm{P} x)
$$

Here the agent knows $x$ is true, and previously knew $x$ had never been true. However, the agent is unsure whether this is the first instance which $x$ has been true. By the perfect recall axiom, in all related runs, all past moments where $x$ was true must be indistinguishable to the agent from the current state.

2) An agent does not know whether time has an end.

$$
\mathrm{L}_{i} \mathrm{GF} \top \wedge \mathrm{L}_{i} \mathrm{FG} \perp
$$

Here, an agent considers two runs possible: one with a final point and one without.

3) An agent may not recognize a final point in time:

$$
\mathrm{G} \perp \wedge \mathrm{L}_{i} \mathrm{GF} \top
$$

This is perhaps a little more challenging. Should an agent recognize when there is no next moment of time? This may vary with context, but from an applications point of view we could imagine an agent monitoring traffic on a communications channel, where the agent is unable to distinguish between no data being transmitted (but the channel silently persisting) the channel closing (a final point in time).

\section{Flows of Time}

All logics we examine satisfy the perfect recall constraint and we define different logics by varying the flows of time $(L,<)$. Recall a linear flow of time $(L,<)$ is defined such that $L$ is any non-empty set, and $<$ is a transitive, irreflexive and anti-symmetric relation such that for all $x, y \in L$, either $x=y, x<y$ or $y<x$. Particularly we consider:

1) General Linear Flows: $\mathcal{T} \mathcal{E} \mathcal{L}_{n}^{p r}$ refers to the case where each run may be any linear flow of time.

2) Uniform Linear Flows: $\mathcal{U} \mathcal{T} \mathcal{E}_{n}^{p r}$ refers to the logic where every run in the model must be over the same flow of time. However, there are no restrictions on what that linear flow of time may be.

3) The Integers: $\mathcal{Z} \mathcal{T} \mathcal{E} \mathcal{L}_{n}^{p r}$ refers to the logic where every run in the a model must be over the integers, $\mathbb{Z}$.

4) The Rationals: $\mathcal{Q} \mathcal{T} \mathcal{E}_{n}^{p r}$ refers to the logic where every run in the a model must be over the rational numbers, Q.

5) The Reals: $\mathcal{R} \mathcal{T} \mathcal{E} \mathcal{L}_{n}^{p r}$ refers to the logic where every run in the a model must be over the integers, $\mathbb{R}$.

\section{COMPLETENESS}

We will give a finite Hilbert-style axiomatization for $\mathcal{T} \mathcal{E} \mathcal{L}_{n}^{p r}$

THEOREM 1: $\mathcal{T} \mathcal{E} \mathcal{L}_{n}^{p r}$ is finitely axiomatizable.

Proof: The axioms are as follows. Besides the axioms for propositional logic, we have the

- axioms for epistemic logic (stating that every $\mathrm{K}_{i}$ is an $S 5$ modality)

- axioms for linear temporal logic (for strict $F$ and P) (see for example [8], Chapter 6).

- $\mathrm{K}_{i} \varphi \rightarrow \mathrm{G}^{\prime} \mathrm{K}_{i} \mathrm{P}^{\prime} \mathrm{K}_{i} \varphi$ (perfect recall axiom, $\mathrm{PR}$ ).

PR says that if agent $i$ knows $\varphi$, then agent $i$ will always know that once (s)he knew $\varphi$. Rules of inference are Modus Ponens and Universal Generalization. We refer to the axiom system as $\mathrm{TEL}_{n}^{p r}$.

First let us note that the above calculus is sound w.r.t. $\mathcal{T E} \mathcal{L}_{n}^{p r}$. We just check the validity of PR. Assume that $(\mathcal{M}, r, k) \models \mathrm{K}_{i} \varphi$ and that $k<l$. We have to show that $(\mathcal{M}, r, l) \models \mathrm{K}_{i} \mathrm{P}^{\prime} \mathrm{K}_{i} \varphi$. So let $r^{\prime}$ and $l^{\prime}$ be such that $r(l) \sim_{i}$ $r^{\prime}\left(l^{\prime}\right)$. We need $\left(\mathcal{M}, r^{\prime}, l^{\prime}\right) \models \mathrm{P}^{\prime} \mathrm{K}_{i} \varphi$. By the perfect recall condition, there is $k^{\prime} \leq l^{\prime}$ with $r(k) \sim_{i} r^{\prime}\left(k^{\prime}\right)$. Hence we have $\left(\mathcal{M}, r^{\prime}, k^{\prime}\right) \models \mathrm{K}_{i} \varphi$, whence $\left(\mathcal{M}, r^{\prime}, l^{\prime}\right) \models \mathrm{P}^{\prime} \mathrm{K}_{i} \varphi$ follows.

To show completeness, assume that $\chi$ is a consistent formula. We will construct a model satisfying $\chi$ in a step-by-step manner by "curing defects". By a partial model $\mathcal{M}$ we mean a subset $M$ of $\mathbb{N} \times \mathbb{Q}$ together with a function $f^{\mathcal{M}}$ associating a maximal consistent set of formulas (mcs, for short) with every element of $M$, and with $n$ equivalence relations $\sim_{i}^{\mathcal{M}}(i \in n)$ such that

1) $(x, y) \quad \sim_{i}^{\mathcal{M}}\left(x^{\prime}, y^{\prime}\right)$ implies that $f^{\mathcal{M}}(x, y)$ and $f^{\mathcal{M}}\left(x^{\prime}, y^{\prime}\right)$ contain the same $\mathrm{K}_{i} \varphi$ formulas,

2) $(x, y),\left(x, y^{\prime}\right) \in M, y<y^{\prime}$ and $\mathrm{G} \varphi \in f^{\mathcal{M}}(x, y)$ imply $\varphi \in f^{\mathcal{M}}\left(x, y^{\prime}\right)$

3) the mirror image of 2 for $\mathrm{H}$. 
We will define four types of defect of a partial model $\mathcal{M}$. Let $(x, y),\left(x^{\prime}, y^{\prime}\right) \in M$ and $\varphi$ be a formula (in the language of $\chi)$ such that $\varphi \in f^{\mathcal{M}}(x, y)$.

Future defect:

$\varphi$ has the form $\mathrm{F} \psi$, and there is no $\left(x, y^{\prime}\right) \in M$ such that $y<y^{\prime}$ and $\varphi \in f^{\mathcal{M}}\left(x, y^{\prime}\right)$.

Past defect:

the mirror image for $P$.

Epistemic defect:

$\varphi$ has the form $\mathrm{L}_{i} \psi$, and there is no $\left(x^{\prime}, y^{\prime}\right) \in M$

\section{Perfect recall defect:} such that $(x, y) \sim_{i}^{\mathcal{M}}\left(x^{\prime}, y^{\prime}\right)$ and $\varphi \in f^{\mathcal{M}}\left(x^{\prime}, y^{\prime}\right)$

$(x, y) \sim_{i}^{\mathcal{M}}\left(x^{\prime}, y^{\prime}\right)$ and $z<y$ such that $(x, z) \in M$ but there is no $z^{\prime} \leq y^{\prime}$ such that $(x, z) \sim_{i}^{\mathcal{M}}\left(x^{\prime}, z^{\prime}\right)$.

Given a countable partial model, there are at most countably many defects. For a structure $X \times Y, d$ is a potential defect if there is a partial model on some $M \subseteq X \times Y$ such that $d$ is a defect. Again, it is not difficult to see that if $X$ and $Y$ are countable, then there are at most countably many potential defects. Thus we can assume that the potential defects of $\mathbb{N} \times \mathbb{Q}$ are enumerated: $D=\left(d_{0}, d_{1}, \ldots\right)$.

Let $\sigma$ be a fair scheduling function of all potential defects of $\mathbb{N} \times \mathbb{Q}$. That is, $\sigma: \omega \rightarrow D$ such that, for every $k$ and $d \in D$, there is $l>k$ such that $\sigma(l)=d$.

We will define the required model for $\chi$ by induction. Assume the following induction hypothesis:

Induction hypothesis: In each step of the construction, we have defined a finite partial model based on a substructure of $\mathbb{N} \times \mathbb{Q}$.

Base step 0: Let $X$ be an arbitrary mcs such that $\chi \in X$. The partial model $\mathcal{M}_{0}$ has universe $\{(0,0)\}, f^{\mathcal{M}_{0}}(0,0)=X$ and, for every $i, \sim_{i}^{\mathcal{M}_{0}}=\{((0,0),(0,0))\}$.

Inductive step $k+1$ : Let the finite partial model defined so far be $\mathcal{M}_{k}$ based on the structure $M_{k} \subseteq N_{k} \times Q_{k}$, where $N_{k} \subset \mathbb{N}$ and $Q_{k} \subset \mathbb{Q}$. Let $\sigma(k)=d$. If $d$ is not a defect of $\mathcal{M}_{k}$, then we define $\mathcal{M}_{k+1}=\mathcal{M}_{k}$. If $d$ is a defect of $\mathcal{M}_{k}$, then we consider the following cases according to the type of the defect.

\section{Future defect:}

We have $(x, y) \in M_{k}$ such that $\mathrm{F} \psi \in f^{\mathcal{M}_{k}}(x, y)=$ $Y$ and, for all $y^{\prime}>y, \psi \notin f^{\mathcal{M}_{k}}\left(x, y^{\prime}\right)$.

By linear temporal logic we can create a witness. First assume that for all $y^{\prime}>y, \mathrm{~F} \psi \in f^{\mathcal{M}_{k}}\left(x, y^{\prime}\right)$. Let $z$ be the largest number such that $(x, z) \in M_{k}, z^{\prime}$ be $z+1$ and $Y^{\prime}$ be a mcs containing $\left\{\varphi: \mathrm{G} \varphi \in f^{\mathcal{M}_{k}}(x, z)\right\} \cup\{\psi\}$.

Otherwise let $z$ be the greatest rational number w.r.t. the following conditions:

- $(x, z) \in M_{k}$,

- $\mathrm{F} \psi \in f^{\mathcal{M}_{k}}(x, z)$.

Let $Z=\left\{\varphi: \mathrm{G} \varphi \in f^{\mathcal{M}_{k}}(x, z)\right\}, Z^{\prime}=\{\rho: \mathrm{H} \rho \in$ $f^{\mathcal{M}_{k}}\left(x, y^{\prime}\right)$ for some $\left.y^{\prime}>z\right\}$ and $Y^{\prime}$ be a mcs containing $Z \cup Z^{\prime} \cup\{\psi\}$. We let $z^{\prime}$ be a rational number greater than $z$ but smaller than any of $\left\{u:(x, u) \in M_{k}, z<u\right\}$.

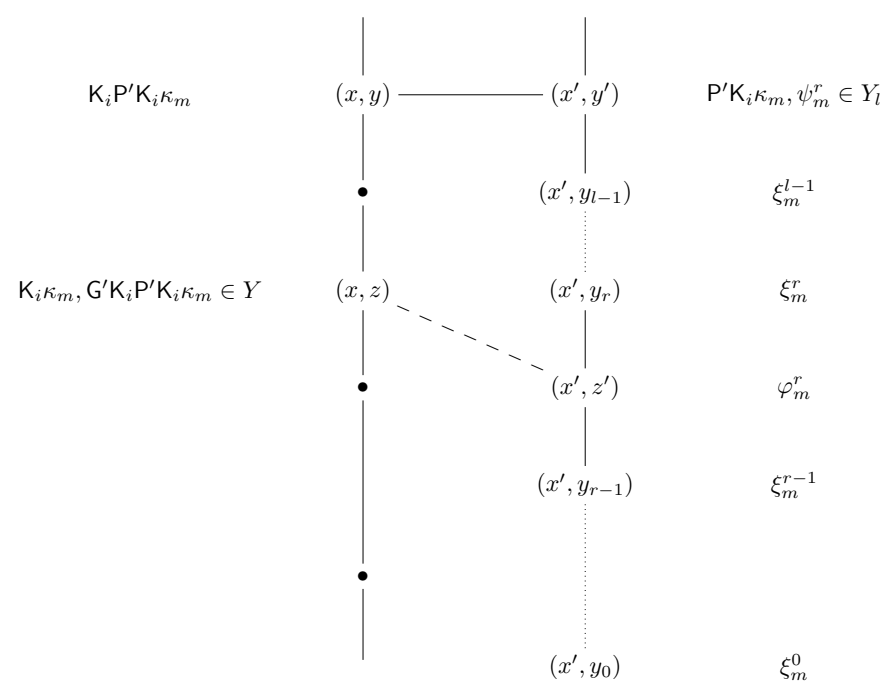

Fig. 1. Curing PR-defect

In both cases the new partial model $\mathcal{M}_{k+1}$ is defined by expanding $\mathcal{M}_{k}$ as $M_{k+1}=M_{k} \cup\left\{\left(x, z^{\prime}\right)\right\}, f^{\mathcal{M}_{k+1}}\left(x, z^{\prime}\right)=$ $Y^{\prime}$ and $\sim_{i}^{\mathcal{M}_{k+1}}=\sim_{i}^{\mathcal{M}_{k}} \cup\left\{\left(\left(x, z^{\prime}\right),\left(x, z^{\prime}\right)\right)\right\}$ for $i \in n$.

Using temporal reasoning, it is easy to check that $\mathcal{M}_{k+1}$ is a partial model.

\section{Past defect:}

This case is completely analogous to the previous one, and we omit the details.

\section{Epistemic defect:}

$\mathrm{L}_{i} \psi \in f^{\mathcal{M}_{k}}(x, y)=Y$, and there is no $\left(x^{\prime}, y^{\prime}\right) \in M_{k}$ such that $(x, y) \sim_{i}^{\mathcal{M}_{k}}\left(x^{\prime}, y^{\prime}\right)$ and $\psi \in f^{\mathcal{M}_{k}}\left(x^{\prime}, y^{\prime}\right)$.

We use epistemic logic to create a witness. By epistemic (in fact, normal modal logic) reasoning, the set $Y^{\prime}=\left\{\mathrm{K}_{i} \varphi\right.$ : $\left.\mathrm{K}_{i} \varphi \in Y\right\} \cup\{\psi\}$ is consistent. Let $z$ be the smallest element of $\mathbb{N}$ such that $z \notin N_{k}$. We define $\mathcal{M}_{k+1}$ as the following expansion of $\mathcal{M}_{k}$ : let $M_{k+1}=M_{k} \cup\{(z, y)\}$, label $(z, y)$ with a mcs $Z \supseteq Y^{\prime}$ and let $\sim_{i}^{\mathcal{M}_{k+1}} \supseteq \sim_{i}^{\mathcal{M}_{k}}$ be the smallest equivalence relation containing $((x, y),(z, y))$, while $\sim_{j}^{\mathcal{M}_{k+1}}=\sim_{j}^{\mathcal{M}_{k}} \cup\{((z, y),(z, y))\}$ for $j \neq i$.

Since $\mathrm{K}_{i}$ is an $S 5$ modality, $\mathcal{M}_{k+1}$ is a partial model.

\section{Perfect recall defect:}

We have $(x, y) \sim_{i}^{\mathcal{M}_{k}}\left(x^{\prime}, y^{\prime}\right)$, and $z<y$ such that $(x, z) \in M_{k}$, but there is no $z^{\prime} \leq y^{\prime}$ such that $(x, z) \sim_{i}^{\mathcal{M}_{k}}\left(x^{\prime}, z^{\prime}\right)$.

We use the perfect recall axiom and temporal reasoning to create a witness, see Figure 1.

We have to find a mcs $Y^{\prime}$ such that $Y^{\prime}$ and $Y=f^{\mathcal{M}_{k}}(x, z)$ agree on $\mathrm{K}_{i}$-formulas, and place $Y^{\prime}$ in the finite linear order in which $\left(x^{\prime}, y^{\prime}\right)$ occurs. Let us consider this linear order $\left(x^{\prime}, y_{0}\right)<\cdots<\left(x^{\prime}, y_{j}\right)$; then $y^{\prime}=y_{l}$ for some $l \leq j$. Let us denote $f^{\mathcal{M}_{k}}\left(x^{\prime}, y_{p}\right)$ by $Y_{p}(0 \leq p \leq l)$.

Our aim is to show that one of the following sets of formulas is consistent.

$\Phi^{0}: \quad\left\{\varphi: \mathrm{H} \varphi \in Y_{l}\right\} \cup\left\{\varphi: \mathrm{H} \varphi \in Y_{l-1}\right\} \cup \cdots \cup\{\varphi: \mathrm{H} \varphi \in$ $\left.Y_{1}\right\} \cup\left\{\varphi: \mathrm{H} \varphi \in Y_{0}\right\} \cup\left\{\mathrm{K}_{i} \varphi: \mathrm{K}_{i} \varphi \in Y\right\}$ 


$$
\begin{array}{ll}
\Phi^{1}: & \left\{\varphi: \mathrm{H} \varphi \in Y_{l}\right\} \cup\left\{\varphi: \mathrm{H} \varphi \in Y_{l-1}\right\} \cup \cdots \cup\{\varphi: \mathrm{H} \varphi \in \\
& \left.Y_{1}\right\} \cup\left\{\mathrm{K}_{i} \varphi: \mathrm{K}_{i} \varphi \in Y\right\} \cup\left\{\varphi: \mathrm{G} \varphi \in Y_{0}\right\} \\
\ldots . & \\
\Phi^{l}: & \left\{\varphi: \mathrm{H} \varphi \in Y_{l}\right\} \cup\left\{\mathrm{K}_{i} \varphi: \mathrm{K}_{i} \varphi \in Y\right\} \cup\{\varphi: \mathrm{G} \varphi \in \\
& \left.Y_{l-1}\right\} \cup \cdots \cup\left\{\varphi: \mathrm{H} \varphi \in Y_{1}\right\} \cup\left\{\varphi: \mathrm{H} \varphi \in Y_{0}\right\}
\end{array}
$$

Let $\left(\mathrm{K}_{i} \varphi_{0}, \ldots, \mathrm{K}_{i} \varphi_{m}, \ldots\right)$ be an enumeration of all the formulas of form $\mathrm{K}_{i} \varphi$ in $Y$, and define $\kappa_{m}=\bigwedge\left\{\mathrm{K}_{i} \varphi_{p}: p \leq m\right\}$. Clearly $\mathrm{K}_{i} \kappa_{m} \leftrightarrow \kappa_{m}$, whence $\mathrm{K}_{i} \kappa_{m} \in Y$. Thus, by the perfect recall axiom, $\mathrm{G}^{\prime} \mathrm{K}_{i} \mathrm{P}^{\prime} \mathrm{K}_{i} \kappa_{m} \in Y$. Since we have a partial model, we get $\mathrm{P}^{\prime} \mathrm{K}_{i} \kappa_{m} \in Y_{l}$. By epistemic reasoning, $\mathrm{P}^{\prime} \kappa_{m} \in Y_{l}$. By our assumption $\kappa_{m} \notin Y_{l}$ for a large enough $m$, whence $\mathrm{P} \kappa_{m} \in Y_{l}$.

For every $p \leq l$, let us enumerate $\left(\varphi_{0}^{p}, \varphi_{1}^{p}, \ldots\right)$ the formulas in $Y_{p}$, and define $\xi_{m}^{p}=\bigwedge\left\{\varphi_{q}^{p}: q \leq m\right\}$. Similarly we define $\gamma_{m}^{p}$ for $\mathrm{G}$-formulas in $Y_{p}$ and $\chi_{m}^{p}$ for $\mathrm{H}$-formulas in $Y_{p}$.

For every $m$ consider the formula $\psi_{m}$ :

$$
\mathrm{P} \kappa_{m} \wedge \xi_{m}^{l} \wedge \mathrm{P}\left(\xi_{m}^{l-1} \wedge \mathrm{P}\left(\xi_{m}^{l-2} \wedge \cdots \wedge \mathrm{P}\left(\xi_{m}^{1} \wedge \mathrm{P} \xi_{m}^{0}\right) \ldots\right)\right)
$$

Since $\mathcal{M}_{k}$ is a partial model, $\psi_{m} \in Y_{l}$, i.e., $\psi_{m}$ is consistent. By our assumption that none of the $Y_{p}$ is the right witness, for a big enough $m, \neg \kappa_{m} \in Y_{p}$ for every $0 \leq p \leq l$, i.e., $\kappa_{m} \wedge \xi_{m}^{p}$ is inconsistent. Then, by temporal reasoning, at least one of the following formulas $\psi_{m}^{p}(0 \leq p \leq l)$ is consistent.

$$
\begin{array}{ll}
\psi_{m}^{0}: & \xi_{m}^{l} \wedge \mathrm{P}\left(\xi_{m}^{l-1} \wedge \mathrm{P}\left(\ldots \wedge \mathrm{P}\left(\xi_{m}^{1} \wedge \mathrm{P}\left(\xi_{m}^{0} \wedge \mathrm{P} \kappa_{m}\right)\right) \ldots\right)\right) \\
\psi_{m}^{1}: & \xi_{m}^{l} \wedge \mathrm{P}\left(\xi_{m}^{l-1} \wedge \mathrm{P}\left(\cdots \wedge \mathrm{P}\left(\xi_{m}^{1} \wedge \mathrm{P}\left(\kappa_{m} \wedge \mathrm{P} \xi_{m}^{0}\right)\right) \ldots\right)\right) \\
\ldots & \\
\psi_{m}^{l}: & \xi_{m}^{l} \wedge \mathrm{P}\left(\kappa_{m} \wedge \mathrm{P}\left(\xi_{m}^{l-1} \wedge \cdots \wedge \mathrm{P}\left(\xi_{m}^{1} \wedge \mathrm{P} \xi_{m}^{0}\right) \ldots\right)\right)
\end{array}
$$

As $m$ grows, for at least one fixed $p$, say $r$, infinitely many formulas $\psi_{m}^{r}$ are consistent. Observe that $\psi_{m^{\prime}}^{p} \rightarrow \psi_{m}^{p}$ for $m^{\prime}>$ $m$, whence $\psi_{m}^{r}$ is consistent for every $m$. Next consider the following formulas $\varphi_{m}^{p}$.

$$
\begin{array}{ll}
\varphi_{m}^{0}: & \chi_{m}^{l} \wedge \chi_{m}^{l-1} \wedge \cdots \wedge \chi_{m}^{1} \wedge \chi_{m}^{0} \wedge \kappa_{m} \\
\varphi_{m}^{1}: & \chi_{m}^{l} \wedge \chi_{m}^{l-1} \wedge \cdots \wedge \chi_{m}^{1} \wedge \kappa_{m} \wedge \gamma_{m}^{0} \\
\cdots & \\
\varphi_{m}^{l}: & \chi_{m}^{l} \wedge \kappa_{m} \wedge \gamma_{m}^{l-1} \wedge \cdots \wedge \gamma_{m}^{1} \wedge \gamma_{m}^{0}
\end{array}
$$

If $\varphi_{m}^{p}$ is inconsistent, then so is the formula $\psi_{m^{\prime}}^{p}$ for a big enough $m^{\prime}$ (by temporal reasoning). Thus $\varphi_{m}^{r}$ must be consistent for all $m$. It follows that $\Phi^{r}$ is consistent.

Then there is a mcs $Z$ containing $\Phi^{r}$. This is the required witness, since we can insert a point $\left(x^{\prime}, z^{\prime}\right)$ into the linear order $\left(x^{\prime}, y_{0}\right)<\cdots<\left(x^{\prime}, y_{l}\right)$ below $\left(x^{\prime}, y_{r}\right)$ (and above $\left(x^{\prime}, y_{r-1}\right)$ if this exists). (Again we use the density of rational numbers here.)

Then the partial model $\mathcal{M}_{k+1}$ is defined by adding $\left(x^{\prime}, z^{\prime}\right)$ to $M_{k}$, labelling it with $Z$, and taking a minimal extension of $\sim_{i}^{\mathcal{M}_{k}}$ so that $(x, z) \sim_{i}^{\mathcal{M}_{k+1}}\left(x^{\prime}, z^{\prime}\right)\left(\right.$ again $\sim_{j}^{\mathcal{M}_{k+1}}=\sim_{j}^{\mathcal{M}_{k}}$ $\cup\left\{\left(\left(x^{\prime}, z^{\prime}\right),\left(x^{\prime}, z^{\prime}\right)\right)\right\}$ for $\left.j \neq i\right)$.

Limit step: We take the union $\mathcal{M}$ of $\mathcal{M}_{k}(k \in \mathbb{N})$. Clearly $\mathcal{M}$ is a partial model. Furthermore, the fair scheduling policy guarantees that it does not contain any defect (once a defect has been cured it cannot reoccur). Thus $\mathcal{M}$ is a partial model of $\chi$ without defects. From $\mathcal{M}$ we can define a model $\mathrm{N}$ for $\chi$ as follows. Let us replace every element $m=(x, y)$ of $\mathcal{M}$ by an $n$-tuple $h(m)=\left(m_{0}, m_{1}, \ldots, m_{n-1}\right)$ such that $h(m)_{i}=$ $h\left(m^{\prime}\right)_{i}$ iff $m \sim_{i} m^{\prime}$ in $\mathcal{M}$. We define $h(m)<h\left(m^{\prime}\right)$ iff

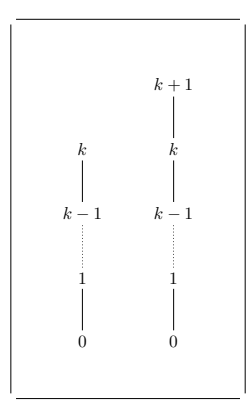

$\mathfrak{F}_{h}$

Fig. 2. Frames $\mathfrak{F}_{k}$ and $\mathfrak{F}_{k+1}$ $m<m^{\prime}$. The valuation $v$ is determined by the labels in $\mathcal{M}$ : $h(m) \in v(p)$ iff $p \in f^{\mathcal{M}}(m)$. It is routine to check that $\mathcal{N}$ indeed satisfies $\chi$.

REMARK 1: The reader might wonder why we are not using the canonical model to satisfy $\chi$. By Sahlqvist's Correspondence and Completeness Theorems (see [2, Theorem 3.54 and Theorem 4.42]) the canonical model satisfies the frame conditions corresponding to our axioms $(<$ is a transitive, non-branching relation, $\sim_{i} \mathrm{~s}$ are equivalence relations and the confluence property of perfect recall holds) and $\chi$. But it is not obvious to us how to turn the canonical model to a model based on tuples of states and runs. Furthermore, our hope is that modifications of the step-by-step construction above might work for special cases (like the reals).

\section{THE UNIFORM CASE}

We start with showing that $\mathcal{U} \mathcal{T} \mathcal{E} \mathcal{L}_{n}$ is not finitely axiomatizable in general. This is in contrast to pure temporal logic where the logic of linear flows is finitely axiomatizable. Later we will see that in specific cases (such as the rationals) uniformity can be achieved.

We prove the theorem below by exploiting the relationship between modal logic and algebraic languages. See [2] (Chapter 5) for a good general overview of this relationship.

THEOREM 2: The uniform version of temporal epistemic $\operatorname{logic} \mathcal{U} \mathcal{T} \mathcal{E} \mathcal{L}_{n}$ is not finitely axiomatizable, even for a single agent $(n=1)$.

Proof: We will prove the theorem by showing that the equational theory of the complex algebras of the frames of $\mathcal{U} \mathcal{T} \mathcal{E} \mathcal{L}_{n}$ is not finitely axiomatizable in first-order logic.

For every positive integer $k$, we let $L_{k}=(k,<)$, i.e., the linear flow of time with length $k-1$. We define the frame $\mathfrak{F}_{k}$ consisting of two disjoint runs, one on $L_{k+1}$ and one on $L_{k+2}$. Thus we can identify these runs with the corresponding flows of time. The relation $\sim_{i}$ is defined as $(k+1 \uplus k+2) \times(k+1 \uplus$ $k+2)$ where $\uplus$ denotes disjoint union. That is, there are two flows of time, with length $k$ and $k+1$, respectively, and the universal relation $\sim_{i}$ to interpret the $i$ th agent's knowledge. Sometimes we will denote the runs $L_{k+1}$ and $L_{k+2}$ of $\mathfrak{F}_{k}$ by $F_{k}^{0}$ and $F_{k}^{1}$, respectively. (See Figure 2). 
Let $\mathfrak{C m}(\mathfrak{F})$ denote the (full) complex algebra of the frame $\mathfrak{F}$. Hence $\mathfrak{C} \mathfrak{m}\left(\mathfrak{F}_{k}\right)$ has universe $\mathcal{P}(k+1 \uplus k+2)$ and operations $\cap, \backslash$ and $o_{\Delta}$ for every modal/temporal connective $\Delta$ :

$$
o_{\Delta}(X)=\left\{y \in k+1 \uplus k+2: y R_{\Delta} x \text { for some } x \in X\right\}
$$

where $R_{\Delta}$ is the accessibility relation defining $\Delta$.

Let $\mathrm{V}$ denote the variety generated by all $\mathfrak{C m}(\mathfrak{F})$ where $\mathfrak{F}$ is a frame of $\mathcal{U} \mathcal{T} \mathcal{E} \mathcal{L}_{n}$. Recall from basic modal logic that the validities of $\mathcal{U} \mathcal{T} \mathcal{E} \mathcal{L}_{n}$ and the valid equations of $\mathrm{V}$ correspond to each other in the following sense:

$$
\mathfrak{F} \models \varphi \leftrightarrow \psi \Longleftrightarrow \mathfrak{C m}(\mathfrak{F}) \models \varphi=\psi
$$

and thus

$$
\mathcal{U} \mathcal{T} \mathcal{E}_{n}=\varphi \leftrightarrow \psi \Longleftrightarrow \mathrm{V} \mid=\varphi=\psi
$$

To prove that $\mathrm{V}$ is not finitely axiomatizable in first-order logic it is enough to show that its complement is not closed under ultraproducts. This is done in the following two lemmas.

LEMMA 1: For every $k, \mathfrak{C m}\left(\mathfrak{F}_{k}\right)$ is not in $\mathrm{V}$.

Proof: Let $\varphi_{k}$ be the formula expressing that time is $k$ long:

$$
\mathrm{H}\left(\mathrm{H} \perp \rightarrow \mathrm{F}^{k}(\top \wedge \mathrm{G} \perp)\right)
$$

and consider $\psi_{k}=\varphi_{k} \rightarrow \mathrm{K} \varphi_{k}$. Note that $\psi_{k}$ is valid formula of $\mathcal{U} \mathcal{T} \mathcal{L}_{n}$, since all runs in a frame have the same length. On the other hand, $\mathfrak{F}_{k} \not \neq \psi_{k}$. Thus $\mathfrak{C} \mathfrak{m}\left(\mathfrak{F}_{k}\right) \not \neq \psi_{k}=\top$, whence $\mathfrak{C} \mathfrak{m}\left(\mathfrak{F}_{k}\right)$ is not in $\mathrm{V}$.

LEMMA 2: Any non-principal ultraproduct $\mathfrak{A}$ of $\mathfrak{C m}\left(\mathfrak{F}_{k}\right)$ is in $\mathrm{V}$.

Proof: Let $\mathfrak{F}$ be the ultraproduct of the frames $\mathfrak{F}_{k}$ over a non-principal ultrafilter $\mathcal{U}$, and denote its complex algebra $\mathfrak{C} \mathfrak{m}(\mathfrak{F})$ by $\mathfrak{B}$. Then the lemma follows by

1) $\mathfrak{B}$ is in $V$,

2) $\mathfrak{A}$ can be embedded into $\mathfrak{B}$.

For 1 observe that $\mathfrak{F}$ has two isomorphic (uncountable) linear flows of time, i.e., it is a frame of the logic. Indeed, one can define the isomorphism by "shifting" (an equivalence class of) a sequence by one to the right. In more detail, let $\bar{x}$ be an element of the ultraproduct of frames and $x$ be such that $\bar{x}=x / \mathcal{U}$ and $\left\{i: x_{i} \in F_{i}^{1}\right\} \in \mathcal{U}$, i.e., $\bar{x}$ is an element of the ultraproduct such that it is defined by a sequence of elements from the runs $F_{i}^{1}$. Since $F_{i}^{1}=F_{i+1}^{0}=(i+2,<)$, for a large set (i.e., in $\mathcal{U}$ ) of indices $i$, we have $x_{i} \in F_{i+1}^{0}$ as well. Let $y=\left(0, x_{0}, x_{1}, \ldots\right)$ and $\bar{y}=y / \mathcal{U}$. We define the isomorphism $\iota$ by $\iota(\bar{x})=\bar{y}$. It is easy to check that $\iota$ is surjective and injective, and that it preserves the ordering $<$.

For 2: This is a standard result. One can define the embedding as follows: given an equivalence class of a sequence $X$ of subsets $X_{i}$, map it to the set of equivalence classes of the sequences determined by the product of the $X_{i}$ s. In more detail, let $\bar{X}$ be an arbitrary element of $A$, and $X_{i}$ be such that $\bar{X}=\prod X_{i} / \mathcal{U}$. Define the isomorphism $\chi$ by

$$
\chi(\bar{X})=\left\{x / \mathcal{U}: x_{i} \in X_{i}\right\} .
$$

It is routine to check that $\chi$ is indeed an isomorphism.
COROLLARY 1: $\mathcal{U} \mathcal{T} \mathcal{E} \mathcal{L}_{n}^{p r}$ is not finitely axiomatizable.

Proof: Note that $\mathfrak{F}$ satisfies the perfect recall condition, since $\sim_{i}$ is the universal relation.

REMARK 2: Since the frame $\mathfrak{F}$ that we defined in the proof of Lemma 2 is discrete, we have that $\mathcal{U} \mathcal{T} \mathcal{E} \mathcal{L}_{n}$ and $\mathcal{U} \mathcal{T} \mathcal{E} \mathcal{L}_{n}^{p r}$ over discrete flows of time are not finitely axiomatizable.

We have shown that temporal epistemic logic with perfect recall cannot be axiomatized with these assumptions alone. However, if we also specify the particular flow of time, then axiomatizations may be feasible.

The axiomatization of $\mathcal{T} \mathcal{E} \mathcal{L}_{n}^{p r}$ and proof presented in Section III is a basic approach to knowledge and time. Effectively we have shown for an arbitrary temporal logic, and an arbitrary epistemic logic, the axiom PR is sufficient to capture the concept of perfect recall. However, practical reasoning about knowledge and time will often use the assumptions that

1) There is one consistent flow of time;

2) this consistent flow of time is common knowledge to all agents.

Below we extend the above axiomatization to apply to such specified uniform flows of time.

\section{THE RATIONALS}

We now suppose the $\mathcal{T} \mathcal{E} \mathcal{L}_{n}^{p r}$ is interpreted solely over flows of time that are isomorphic to the rational numbers. To axiomatize such a logic it is sufficient to add to the axioms above the no-end-point axiom (NEP) and the density axiom (Dense):

$$
\begin{array}{cl}
\text { NEP } & \mathrm{P} \top \wedge \mathrm{F} \top \\
\text { Dense } & \mathrm{F} p \rightarrow \mathrm{FF} p .
\end{array}
$$

We let QTEL $_{n}^{p r}$ be the system TEL ${ }_{n}^{p r}$ augmented with NEP and Dense.

THEOREM 3: The system QTEL $_{n}^{p r}$ is sound and complete for temporal epistemic logic with perfect recall over rational flows of time, $\mathcal{Q T} \mathcal{E} \mathcal{L}_{n}^{p r}$.

Proof: Soundness is easy to check and is left to the reader.

To prove completeness we show that the construction above can be extended to include and cure density defects. That is, we apply the construction for general linear flows of time (above) with a new density defect in the schedule and suppose each mcs is consistent with respect to all substitution instances of the axioms NEP and Dense. (Note that the end points will not require a new defect since an $\mathrm{F} T$ defect will be cured as a future defect). In more detail, we call $\left(x, y, y^{\prime}\right)$ a density defect if $y<y^{\prime}, f^{\mathcal{M}_{k}}(x, y)=\Psi, f^{\mathcal{M}_{k}}\left(x, y^{\prime}\right)=\Phi$, and for all $z$ with $y<z<y^{\prime}, f^{\mathcal{M}_{k}}(x, z)$ is undefined. We include all density defects to the schedule of defects waiting to be cured.

Given a density defect as above, we have for all $\alpha \in \Phi$, $\mathrm{F} \alpha \in \Psi$. By the density axiom it follows that $\mathrm{FF} \alpha \in \Psi$. Therefore, for all $\alpha \in \Phi$ and $\beta \in \Psi, \beta \wedge \mathrm{FF} \alpha$ is consistent. Hence $\mathrm{P} \beta \wedge \mathrm{F} \alpha$ is consistent by temporal reasoning. It follows that there is a mcs $\Lambda$ such that for all $\beta \in \Psi, \mathrm{P} \beta \in \Lambda$ and for all $\alpha \in \Phi, \mathrm{F} \alpha \in \Lambda$. If such a $\Lambda$ could not be found, there must be some finite $\alpha \in \Phi$ and $\beta \in \Psi$ such that $\mathrm{F} \alpha \rightarrow \neg \mathrm{P} \beta$ contradicting the reasoning above. 
Thus we can define $\mathcal{M}_{k+1}=\mathcal{M}_{k} \cup\{(x, z)\}$ and let $f^{\mathcal{M}_{k+1}}(x, z)=\Lambda$ and $\sim_{i}^{\mathcal{M}_{k+1}}=\sim_{i}^{\mathcal{M}_{k}} \cup\{((x, z),(x, z))\}$ for $i \in n$, curing the defect.

\section{THE INTEGERS}

Previously temporal epistemic logic with perfect recall, next and until, but without past operators has been axiomatized over integer flows of time [9], [10]. Also, temporal epistemic logic with perfect recall, next, until and since has been axiomatized over flows of time isomorphic to the natural numbers [11]. For completeness we examine the temporal epistemic logic with perfect recall using only the tense operators (so that next and previous operators are not available). Note that the axiomatizations in [9], [10] make use of the operators until and next which are known to be expressively complete for the natural numbers, whereas $\mathrm{F}$ and $\mathrm{P}$ are not expressively complete). Furthermore, the axiomatization relies on the relationship between the next, until and knowledge operators to capture the perfect recall property. For these reasons the axiomatization of $\mathrm{ZTEL}_{n}^{p r}$ does not follow trivially from the works mentioned above.

An axiomatization for temporal epistemic logic over the integers can be defined by adding the axioms NEP (see above), and:

$$
\begin{array}{ll}
\text { ZF } & (\mathrm{G}(\mathrm{G} p \rightarrow p)) \rightarrow(\mathrm{FG} p \rightarrow \mathrm{G} p) \text { discrete future } \\
\mathbf{Z P} & (\mathrm{H}(\mathrm{H} p \rightarrow p)) \rightarrow \mathrm{PH} p \rightarrow \mathrm{H} p) \text { discrete past }
\end{array}
$$

These axioms are known to be sufficient to axiomatize the tense operators, $\mathrm{F}$ and $\mathrm{P}$, over the integers (see for example [1]). We define $\mathrm{ZTEL}_{n}^{p r}$ to be the axiom system $\mathrm{TEL}_{n}^{p r}$ augmented with the axioms NEP, ZF and ZP.

LEMMA 3: The system $\mathrm{ZTEL}_{n}^{p r}$ is sound for temporal epistemic logics with perfect recall over integer flows of time.

Proof: (Sketch) The soundness of NEP, ZF and $\mathbf{Z P}$ is well known (e.g. [1]). The soundness of the other axioms follows from their soundness for general linear flows of time.

We conjecture that the system is also complete, but the proof has thus far been elusive. The approach taken has been to to show that every formula that is consistent with $\mathrm{ZTEL}_{n}^{p r}$ has an integer model. We know that every formula consistent with $\mathrm{ZTEL}_{n}^{p r}$ is consistent with $\mathrm{TEL}_{n}^{p r}$ (the logic of general linear flows), so we are able to apply Theorem 1 to build a model that satisfies both the consistent formula, as well as every substitution instance of the axioms NEP, ZF and ZP. Having built this model, we attempt to transform it into an integer model without introducing any new defects, using the techniques of [13]. While the strategy is promising, there remain some anomalous cases which are difficult to address.

CONJECTURE 1: The system $\mathrm{ZTEL}_{n}^{p r}$ is complete for temporal epistemic logics with perfect recall over integer flows of time.

\section{THE REALS}

We now present an axiomatization for logics of knowledge with perfect recall over real flows of time. For notational convenience, we only present the single agent case, but the multi-agent case may be treated similarly.

As with the previous results, we simply augment the axiom systems for general flows of time with sufficient axioms to ensure that all the legitimate flows of time will be isomorphic to the real line. We show that, as with linear temporal logic, it is sufficient to augment the axioms for rational flows of time (i.e. density axioms and no end-points) with an axiom for Dedekind completeness. In terms of topology of the line, these axioms ensure that every convergent sequence of points converges to some accumulation point.

The Dedekind axiom is:

$$
\text { D } \quad \mathrm{FH} p \rightarrow(\mathrm{G} p \vee \mathrm{F}(\mathrm{H} p \wedge \neg p) \vee \mathrm{F}(\mathrm{H} p \wedge p \wedge \mathrm{GP} \neg p))
$$

and we let RTEL $_{n}^{p r}$ be the axiom system QTEL ${ }_{n}^{p r}$ augmented with the axiom D. (Note that as we are extending QTEL $_{n}^{p r}$, density and no end-points are already given).

Note that the inverted version of $\mathbf{D}$, below, can be inferred using $\mathbf{D}$ and the other temporal axioms presented.

$$
\mathrm{PG} p \rightarrow(\mathrm{H} p \vee \mathrm{P}(\mathrm{G} p \wedge \neg p) \vee \mathrm{P}(\mathrm{G} p \wedge p \wedge \mathrm{HF} \neg p)
$$

LEMMA 4: The system $\mathrm{RTEL}_{n}^{p r}$ is sound for temporal epistemic logics with perfect recall over real flows of time.

The proof is relatively straightforward and is left to the reader.

CONJECTURE 2: The system RTEL ${ }_{n}^{p r}$ is complete for temporal epistemic logics with perfect recall over real flows of time.

As with the integer case, we attempt to show every formula that is consistent with $\mathrm{RTEL}_{n}^{p r}$ has a model over real-flows of time via a rational-flowed model for that formula. Given a rational-flowed model of the consistent formula we attempt to convert that model into a real-flowed model without introducing any additional defects. It was hoped that we could extend the knowledge relations and the valuation of atomic propositions to include the irrational points, as can be done in the purely temporal case (see for example [7]). However, in the presence of epistemic operators with perfect recall this is not straightforward, and it appears that this technique needs to be further extended.

\section{CONCLUSION}

Here we have presented a number of axiomatizations and related results for temporal epistemic logics with perfect recall and varying flows of time. We have shown the axiomatizations for $\mathcal{T} \mathcal{E} \mathcal{L}_{n}^{p r}$ (where flows of time are arbitrary) and $\mathcal{Q T} \mathcal{E} \mathcal{L}_{n}^{p r}$ (where the flows of time are isomorphic to the rational numbers) are sound and complete. We have also shown that the logic $\mathcal{U} \mathcal{T} \mathcal{E} \mathcal{L}_{1}$ (where the flow of time is arbitrary, but known to all agents) no finite axiomatization can be given. In the remaining cases, $\mathcal{Z} \mathcal{T} \mathcal{E} \mathcal{L}_{n}^{p r}$ (the integers) and $\mathcal{R} \mathcal{T} \mathcal{E} \mathcal{L}_{n}^{p r}$ (the reals) work is ongoing. We propose sound axiomatizations here, and are working towards completeness proofs. 


\section{REFERENCES}

[1] J. van Benthem. The Logic of Time. Kluwer Academic Publishers, 1983.

[2] Patrick Blackburn, Maarteb de Rijke, and Yde Venema. Modal Logic. Cambridge University Press, 2001.

[3] J. M. Davoren and Anil Nerode. Logics for hybrid systems. In Proceedings of the IEEE. Springer-Verlag, 1993.

[4] R. Fagin, J. Halpern, Y. Moses, and M. Vardi. Reasoning about knowledge. MIT Press, 1995.

[5] T. French, R. van der Men, and M. Reynolds. Axioms for logics of knowledge and past time: synchrony and unique initial states. Advances in Modal Logic, 5:53-72, 2005.

[6] D. Gabbay, A. Kurucz, F. Wolter, and M. Zakharayashev. Many Dimensional Modal Logics: Theory and Applications. Elsevier, 2003.

[7] Dov Gabbay and Ian Hodkinson. An axiomatization of until and since over the real numbers. Journal of Logic and Computation, 1:229-260, 1990.

[8] R. Goldblatt. Logics of Time and Computation. Center for the Study of Language and Information, 1987.
[9] J. Halpern, R. van der Meyden, and M. Vardi. Complete axiomatizations for reasoning about knowledge and time. SIAM Journal on Computing, pages 674-703, 2004.

[10] R. van der Meyden. Axioms for knowledge and time in distributed systems with perfect recall. In Logic in Computer Science, pages 448457, 1994.

[11] R. van der Meyden, 2002. Manuscript

[12] A. Pnueli. The temporal logic of programs. In Proceedings of the Eighteenth Symposium on Foundations of Computer Science, pages 4657, 1977.

[13] Mark Reynolds. Axiomatizing until and since over integer time. In D. Gabbay and H.-J. Ohlbach, editors, Lecture Note in A.I., volume 827, pages 117-132, 1994

[14] Mark Reynolds. The complexity of temporal logic over the reals. Research Report cs.LO/9910012, arXiv, October 1999. 